\title{
Exact Solutions and Light Bullet Soliton Solutions of the Two-Dimensional Generalized Nonlinear Schrödinger Equation with Distributed Coefficients
}

\author{
W.-H. HuAnG*, C.-F. Mu, Y. Xu And B.-G. Ding \\ School of Science, Huzhou Teachers College, Huzhou 313000, China \\ (Received July 22, 2012; in final form July 23, 2013)
}

\begin{abstract}
A generalized $\frac{G^{\prime}}{G}$-expansion method is extended to construct exact solutions to the two-dimensional generalized nonlinear Schrödinger equation with distributed coefficients. Hyperbolic function solution, trigonometric function solution and rational exact solution with parameters are obtained. Selecting parameters and parameter functions properly, novel light bullet soliton solutions with or without the chirp are presented.
\end{abstract}

DOI: 10.12693 /APhysPolA.124.622

PACS: 02.30.Jr, 03.65.Ge, 05.45.Yv, 42.65.Tg

\section{Introduction}

The nonlinear Schrödinger equation (NLSE) is one of the most important universal nonlinear models that naturally arise in many branches of physics, such as nonlinear optics, condensed mater physics, Bose-Einstein condensates, plasma physics and hydrodynamics [1]. It is integrable in $1+1$ dimensions $[(1+1) \mathrm{D}][2,3]$, with many stable soliton solutions report so far. The one-dimensional NLSE with distributed coefficients contains exact tanh-shaped and sech-shaped stable solution pulses as solitons $[4,5]$. These soliton pulses exhibit linear chirp and possess huge applicative potential in optical communication technology [6]. On the other hand, it is shown a large class of linear equations admit of "soliton-like" solutions and mutatis mutandis, localized solutions exist even for ordinary (linear) Schrödinger equation within standard quantum mechanics [7]. Recently, great interest has been generated when it was suggested that two-dimensional NLSE with distributed coefficients may lead to stable $2 \mathrm{D}$ solitons [8]. The stabilizing mechanism was the sign-alternating Kerr nonlinearity in a layered medium. A vigorous search for stabilized periodic solutions of the 2D NLSE with distributed coefficients has been launched $[9,10]$; however, out of necessity, it has been numerical. Very recently, exact analytical spatial soliton solutions of the 2D NLSE with distributed coefficients are also found by Zhong et al. [11] utilizing the F-expansion method [12]. The goal of the present work is to conduct further investigations of the 2D NLSE by the so-called $\frac{G^{\prime}}{G}$-expansion method. The $\frac{G^{\prime}}{G}$-expansion method is first proposed by Wang et al. [13] recently, and then developed and generalized to seek travelling wave solutions for constant-coefficient and variable-coefficient

*corresponding author; e-mail: whhuanghz@hutc.zj.cn nonlinear physical models [14-16]. In this letter, we extend the generalized $\frac{G^{\prime}}{G}$-expansion method [15] to consider the generalized $2 \mathrm{D}$ NLSE with distributed coefficients, many types of exact analytical solutions and even the light bullet soliton solutions are obtained.

\section{The generalized $\left(\frac{G^{\prime}}{G}\right)$-expand method}

First, we give a brief description for the generalized $\left(\frac{G^{\prime}}{G}\right)$-expand method. For a given nonlinear evolution equation (NLEE) with independent variables $X=$ $(x, y, z, \ldots, t)$ and dependent variable $u$ :

$$
\begin{gathered}
F\left(u, u_{t}, u_{x}, u_{y}, u_{z}, \ldots, u_{x t}, u_{y t}, u_{z t},\right. \\
\left.\quad \ldots, u_{t t}, u_{x x}, u_{y y}, u_{z z}, \ldots\right)=0,
\end{gathered}
$$

we suppose its solution can be expressed by a polynomial in $\left(\frac{G^{\prime}}{G}\right)$ as follows:

$$
u=\sum_{i=1}^{m} \alpha_{i}(X)\left(\frac{G^{\prime}}{G}\right)^{i}+\alpha_{0}(X), \quad \alpha_{m}(X) \neq 0,
$$

where $\alpha_{0}(X), \alpha_{i}(X)(i=1,2, \ldots, m)$ and $\xi=\xi(X)$ are all functions of $X$ to be determined later, $G=G(\xi)$ satisfies a second order linear ordinary differential equation

$$
G^{\prime \prime}+\lambda G^{\prime}+\mu G=0,
$$

where $G^{\prime \prime}=\frac{\partial^{2} G(\xi)}{\partial \xi^{2}}, G^{\prime}=\frac{\partial G(\xi)}{\partial \xi}$, and $\lambda, \mu$ are real constants.

To determine $u$ explicitly, we take the following four steps:

Step1. Determine the integer $m$ by balancing the highest order nonlinear terms and the highest order partial derivative of $u$ in Eq. (1).

Step 2. Substitute (2) along with Eq. (3) into Eq. (1) and collect all terms with the same order of $\left(\frac{G^{\prime}}{G}\right)$ together, the left hand side of Eq. (1) was converted into a polynomial in $\left(\frac{G^{\prime}}{G}\right)$. Then set each coefficient of this polynomial to zero to derive a set of over-determined partial differential equations for $\alpha_{0}(X), \alpha_{i}(X)$ and $\xi$. 
Step 3. Solve the system of over-determined partial differential equations obtained in step 2 for $\alpha_{0}(X), \alpha_{i}(X)$ and $\xi$ by use of Mathematica or Maple.

Step 4. Use the results obtained in above steps to derive a series of fundamental solutions of Eq. (3) depending on $\left(\frac{G^{\prime}}{G}\right)$, since the solutions of Eq. (3) have been well known for us, then we can obtain exact solutions of Eq. (1).

\section{Exact solutions for the generalized 2D NLSE}

In Cartesian coordinates, the generalized NLSE describing the propagation of $2 \mathrm{D}$ spatial solitons in bulk optical media with varying coefficients is expressed as [17]:

$$
\mathrm{i} \frac{\partial u}{\partial z}+\frac{1}{2} \beta(z)\left(\frac{\partial^{2}}{\partial x^{2}}+\frac{\partial^{2}}{\partial y^{2}}\right) u+\chi(z)|u|^{2} u=\mathrm{i} \gamma(z) u
$$

where $u(z, x, y)$ is the complex envelope of the electrical field in the moving frame, $z$ is the normalized distance of propagation, and $x$ and $y$ are the normalized coordinates in the transverse plane. The function $\beta(z)$ represents the dispersion coefficient, $\chi(z)$ the nonlinearity coefficient, and $\gamma(z)$ the gain $(\gamma>0)$ or the loss $(\gamma<0)$ coefficient. Thus, Eq. (4) describes the propagation behavior of an optical beam in a Kerr-like medium with varying dispersion, nonlinearity, and gain or loss. We define the complex $u$ field as:

$$
u(z, x, y)=A(z, x, y) \mathrm{e}^{\mathrm{i} B(z, x, y)},
$$

where $A(z, x, y)$ and $B(z, x, y)$ are real functions. Substituting $u(z, x, y)$ into Eq. (4), we find the following coupled equations for the phase $B(z, x, y)$ and the amplitude $A(z, x, y)$ :

$$
\begin{aligned}
& \frac{\partial A}{\partial z}+\frac{1}{2} \beta(z)\left(2 \frac{\partial A}{\partial x} \frac{\partial B}{\partial x}+2 \frac{\partial A}{\partial y} \frac{\partial B}{\partial y}\right. \\
& \left.+A \frac{\partial^{2} B}{\partial^{2} x^{2}}+A \frac{\partial^{2} B}{\partial^{2} y^{2}}\right)-\gamma(z) A=0, \\
& -A \frac{\partial B}{\partial z}+\frac{1}{2} \beta(z)\left[\frac{\partial^{2} A}{\partial^{2} x^{2}}+\frac{\partial^{2} A}{\partial^{2} y^{2}}\right. \\
& \left.-A\left(\frac{\partial B}{\partial x}\right)^{2}-A\left(\frac{\partial B}{\partial y}\right)^{2}\right]+\chi(z) A^{3}=0 .
\end{aligned}
$$

According to the balance principle and the generalized $\frac{G^{\prime}}{G}$-expansion method [14], the solution of Eqs. (6) and $(7)$ can be expressed in the following form:

$$
\begin{aligned}
& A(z, x, y)=f_{0}+f_{1} \frac{G(\xi)^{\prime}}{G(\xi)}, \\
& \xi=k(z) x+l(z) y+\omega(z), \\
& B(z, x, y)=a(z)\left(x^{2}+y^{2}\right)+b(z)(x+y)+e(z),
\end{aligned}
$$

where $G=G(\xi)$ satisfies Eq. (3) and $f_{0}, f_{1}, k, l, \omega$, $a, b, e$ are the parameters to be determined. In this quadratic form of Eq. (9), the parameter $a(z)$ is related to the wave front curvature; it is a measure of the phase chirp imposed on the solitary wave. Substituting Eqs. (8) and (9) into Eqs. (6) and (7) and collecting all the terms with the same order of $\frac{G^{\prime}}{G}$ together, the left sides of Eqs. (6) and (7) are converted into two polynomials of $x^{i_{1}} y^{j_{1}}\left(\frac{G^{\prime}}{G}\right)^{k_{1}}\left(i_{1}=0,1 ; j_{1}=0,1 ; k_{1}=0,1,2\right)$ and $x^{i_{2}} y^{j_{2}}\left(\frac{G^{\prime}}{G}\right)^{k_{2}}\left(i_{2}=0,1,2 ; j_{2}=0,1,2 ; k_{2}=0,1,2,3\right)$, respectively. Setting each coefficient of the two polynomials to zero, after having simplified one can finally derive a system of algebraic or first-order ordinary differential equations for $f_{0}, f_{1}, k, l, \omega, a, b$, and $e$ as follows:

$$
\begin{aligned}
& -f_{1} \frac{\partial \omega}{\partial z} \mu+2 \beta f_{0} a-\gamma f_{0}+\frac{\partial f_{0}}{\partial z} \\
& \quad-\beta f_{1} l b \mu-\beta f_{1} k b \mu=0, \\
& -f_{1} p\left(\frac{\partial k}{\partial z}+2 \beta k a\right)=0, \\
& -f_{1} p\left(\frac{\partial l}{\partial z}+2 \beta l a\right)=0, \\
& -f_{1}\left(\frac{\partial \omega}{\partial z}+\beta k b+\beta l b\right)=0, \\
& -f_{j}\left(\frac{\partial a}{\partial z}+2 \beta a^{2}\right)=0, \\
& -f_{j}\left(\frac{\partial b}{\partial z}+2 \beta a b\right)=0, \\
& \frac{\partial f_{1}}{\partial z}+2 \beta f_{1} a-\gamma f_{1}-\beta f_{1} k b \lambda-f_{1} \frac{\partial \omega}{\partial z} \lambda-\beta f_{1} l b \lambda=0 \\
& \frac{3}{2} f_{1}\left(\beta l^{2} \lambda+\beta k^{2} \lambda+2 \chi f_{0} f_{1}\right)=0, \\
& f_{1}\left(\chi f_{1}^{2}+\beta l^{2}+\beta k^{2}\right)=0, \\
& \frac{1}{2} f_{1}\left(\beta l^{2} \lambda^{2}-2 \frac{\partial e}{\partial z}+2 \beta l^{2} \mu+2 \beta k^{2} \mu-2 \beta b^{2}\right.
\end{aligned}
$$

where $p=1, \lambda, \mu$ and $j=0,1$.

Solving Eqs. (10)-(20) self-consistently, we have a set of conditions on the coefficients and parameters as follows:

$$
\begin{aligned}
& f_{0}=f_{10} \exp \left(\int_{0}^{z} \gamma-2 \beta a_{0} \alpha \mathrm{d} z\right), \\
& f_{1}=\frac{2 f_{10}}{\lambda} \exp \left(\int_{0}^{z} \gamma-2 \beta a_{0} \alpha \mathrm{d} z\right), \\
& a=a_{0} \alpha, \quad b=b_{0} \alpha, \quad k=k_{0} \alpha, \quad l=l_{0} \alpha, \\
& \omega=\omega_{0}-\alpha\left(k_{0}+l_{0}\right) b_{0} \int_{0}^{z} \beta \mathrm{d} z, \\
& e=\left[\left(k_{0}^{2}+l_{0}^{2}\right)\left(\mu-\frac{1}{4} \lambda^{2}\right)-b_{0}^{2}\right] \int_{0}^{z} \beta \alpha^{2} \mathrm{~d} z,
\end{aligned}
$$

where $f_{10}, a_{0}, b_{0}, k_{0}, l_{0}, \omega_{0}$ are real constants, and $\alpha=\left[1+2 a_{0} \int_{0}^{z} \beta \mathrm{d} z\right]^{-1}$ is the normalized chirp function. The chirp function $\alpha$ is related only to dispersion coefficients; however, it affects all of the parameters. In the 
case when there is no chirp, $a_{0}=0$ and $\alpha=1$, the parameters $k, l$, and $b$ are all constants. In the presence of chirp, they all acquire the prescribed $z$ dependence. The chirp also influence the form of the amplitude $A$ through the dependence of $f_{0}, f_{1}$ and $\xi$ on $\alpha$. It should also be noted that $\chi$ is not arbitrary but depends on $\alpha, \beta$ and $\gamma$ :

$$
\chi=-\frac{\beta \lambda^{2} \alpha^{2}}{4 f_{10}^{2}}\left(k_{0}^{2}+l_{0}^{2}\right) \exp \left(-2 \int_{0}^{z} \gamma-2 \beta a_{0} \alpha \mathrm{d} z\right) \text {. }
$$

Hence, the solutions found can exist only under certain conditions and the system parameter functions $\beta(z), \gamma(z)$ and $\chi(z)$ cannot be all chosen independently, i.e., the functions $\beta(z), \gamma(z)$ are chosen as independent while the nonlinearity coefficient $\chi(z)$ is determined by Eq. (25). Substituting Eqs. (8) and (9) together with Eqs. (21)-(24) into Eq. (5), we have the fundamental solution of Eq. (4):

$$
\begin{aligned}
u= & f_{10} \exp \left(\int_{0}^{z} \gamma-2 \beta a_{0} \alpha \mathrm{d} z\right)\left[1+\frac{2}{\lambda}\left(\frac{G^{\prime}}{G}\right)\right] \\
& \times \exp \left(\mathrm { i } \left[a_{0} \alpha\left(x^{2}+y^{2}\right)+b_{0} \alpha(x+y)\right.\right. \\
& \left.\left.+\left(\left(k_{0}^{2}+l_{0}^{2}\right)\left(\mu-\frac{1}{4} \lambda^{2}\right)-b_{0}^{2}\right) \int_{0}^{z} \beta \alpha^{2} \mathrm{~d} z\right]\right),
\end{aligned}
$$

where $G=G(\xi), \xi=k_{0} \alpha x+l_{0} \alpha y+\omega_{0}-\alpha\left(k_{0}+\right.$ $\left.l_{0}\right) b_{0} \int_{0}^{z} \beta \mathrm{d} z$, and $\alpha=\left[1+2 a_{0} \int_{0}^{z} \beta \mathrm{d} z\right]^{-1}$.

Substituting the general solutions of Eq. (3) into Eq. (26), we have three types of exact solutions of $2 \mathrm{D}$ NLSE as follows:

When $\lambda^{2}-4 \mu>0$, we obtain the hyperbolic function solution

$$
\begin{gathered}
u_{1}=f_{10} \exp \left(\int_{0}^{z} \gamma-2 \beta a_{0} \alpha \mathrm{d} z\right) \\
{\left[1+\frac{2}{\lambda} \frac{C_{1} \sinh \left(\frac{\sqrt{\lambda^{2}-4 \mu}}{2} \xi\right)+C_{2} \cosh \left(\frac{\sqrt{\lambda^{2}-4 \mu}}{2} \xi\right)}{\left.C_{1} \cosh \left(\frac{\sqrt{\lambda^{2}-4 \mu}}{2} \xi\right)+C_{2} \sinh \left(\frac{\sqrt{\lambda^{2}-4 \mu}}{2} \xi\right)\right]}\right.} \\
\quad \times \exp \left(\mathrm { i } \left[a_{0} \alpha\left(x^{2}+y^{2}\right)+b_{0} \alpha(x+y)\right.\right. \\
\left.\left.+\left(\left(k_{0}^{2}+l_{0}^{2}\right)\left(\mu-\frac{1}{4} \lambda^{2}\right)-b_{0}^{2}\right) \int_{0}^{z} \beta \alpha^{2} \mathrm{~d} z\right]\right),
\end{gathered}
$$

where $\xi=k_{0} \alpha x+l_{0} \alpha y+\omega_{0}-\alpha\left(k_{0}+l_{0}\right) b_{0} \int_{0}^{z} \beta \mathrm{d} z, \alpha=$ $\left[1+2 a_{0} \int_{0}^{z} \beta \mathrm{d} z\right]^{-1}$, and $C_{1}, C_{2}$ are arbitrary constants.

When $\lambda^{2}-4 \mu<0$, we have the trigonometric function solution

$$
\begin{gathered}
u_{2}=f_{10} \exp \left(\int_{0}^{z} \gamma-2 \beta a_{0} \alpha \mathrm{d} z\right) \\
{\left[1+\frac{2}{\lambda} \frac{-C_{1} \sin \left(\frac{\sqrt{4 \mu-\lambda^{2}}}{2} \xi\right)+C_{2} \cos \left(\frac{\sqrt{4 \mu-\lambda^{2}}}{2} \xi\right)}{C_{1} \cos \left(\frac{\sqrt{4 \mu-\lambda^{2}}}{2} \xi\right)+C_{2} \sin \left(\frac{\sqrt{4 \mu-\lambda^{2}}}{2} \xi\right)}\right]} \\
\quad \times \exp \left(\mathrm { i } \left[a_{0} \alpha\left(x^{2}+y^{2}\right)+b_{0} \alpha(x+y)\right.\right.
\end{gathered}
$$

$$
\left.\left.+\left(\left(k_{0}^{2}+l_{0}^{2}\right)\left(\mu-\frac{1}{4} \lambda^{2}\right)-b_{0}^{2}\right) \int_{0}^{z} \beta \alpha^{2} \mathrm{~d} z\right]\right),
$$

where $\xi=k_{0} \alpha x+l_{0} \alpha y+\omega_{0}-\alpha\left(k_{0}+l_{0}\right) b_{0} \int_{0}^{z} \beta \mathrm{d} z, \alpha=$ $\left[1+2 a_{0} \int_{0}^{z} \beta \mathrm{d} z\right]^{-1}$, and $C_{1}, C_{2}$ are arbitrary constants.

When $\lambda^{2}-4 \mu=0$, we get the rational solution

$$
\begin{aligned}
u_{3} & =f_{10} \exp \left(\int_{0}^{z} \gamma-2 \beta a_{0} \alpha \mathrm{d} z\right) \\
& \times\left(1+\frac{2}{\lambda} \frac{C_{2}}{C_{1}+C_{2} \xi}\right) \\
& \times \exp \left(\mathrm { i } \left[a_{0} \alpha\left(x^{2}+y^{2}\right)+b_{0} \alpha(x+y)\right.\right. \\
& \left.\left.+\left(\left(k_{0}^{2}+l_{0}^{2}\right)\left(\mu-\frac{1}{4} \lambda^{2}\right)-b_{0}^{2}\right) \int_{0}^{z} \beta \alpha^{2} \mathrm{~d} z\right]\right),
\end{aligned}
$$

where $\xi=k_{0} \alpha x+l_{0} \alpha y+\omega_{0}-\alpha\left(k_{0}+l_{0}\right) b_{0} \int_{0}^{z} \beta \mathrm{d} z, \alpha=$ $\left[1+2 a_{0} \int_{0}^{z} \beta \mathrm{d} z\right]^{-1}$, and $C_{1}, C_{2}$ are arbitrary constants.

\section{Light bullet soliton solutions for the generalized $2 \mathrm{D}$ NLSE}

For the solutions of Eq. (4) above, due to the fact that the arbitrary functions $\gamma(z)$ and $\beta(z)(\chi(z)$ depend on $\gamma(z)$ and $\beta(z)$ by Eq. (25)), we have freedom in selecting these functions appropriately according to the actual physical requirements. Thus we can construct abundant periodic wave solutions and solitary wave solutions. Here we only consider a simple special case. For the solution $u_{1}$ expressed in Eq. (27), when $C_{2}=0$, and $\mu=\frac{\lambda^{2}-4 R}{4}$, we have

$$
\begin{aligned}
u_{4} & =f_{10} \exp \left(\int_{0}^{z} \gamma-2 \beta a_{0} \alpha \mathrm{d} z\right)\left[1+\frac{2}{\lambda} \tanh (\sqrt{R \xi})\right] \\
& \times \exp \left(\mathrm { i } \left[a_{0} \alpha\left(x^{2}+y^{2}\right)+b_{0} \alpha(x+y)\right.\right. \\
& \left.\left.-\left(\left(k_{0}^{2}+l_{0}^{2}\right) R+b_{0}^{2}\right) \int_{0}^{z} \beta \alpha^{2} \mathrm{~d} z\right]\right),
\end{aligned}
$$

where $\xi=k_{0} \alpha x+l_{0} \alpha y+\omega_{0}-\alpha\left(k_{0}+l_{0}\right) b_{0} \int_{0}^{z} \beta \mathrm{d} z, R>0$ and $\chi$ satisfies Eq. (25). Taking the dispersion coefficient $\beta$ to be of the form $\beta=\beta_{0}+k_{a} z$ and the gain (loss) coefficient $\gamma$ to be a small constant, we obtain light bullet soliton solutions of the 2D NLSE, which are depicted in detail in Fig. 1a and b, with and without the chirp. In Fig. 1a, b, the intensity $U=\left|u_{4}\right|^{2}$ determined by Eq. (30) are plotted and the functions of the propagation distance are presented as functions of $X=k_{0} x+l_{0} y$ and $z$. Similarly, if the dispersion coefficient $\beta$ is fixed as periodic function $\beta=\beta_{0} \cos \left(k_{b} z\right)$, then the special type of light bullet solitons are shown in Fig. $2 \mathrm{a}$ and b. One can see the effect of the particular periodic chirp function determined by the dispersion coefficient $\beta=\beta_{0} \cos \left(k_{b} z\right)$ which is the production of a periodic variation along the propagation direction ( $z$ direction) and a monotonic asymmetric change in the transverse direction ( $X$ direction). 


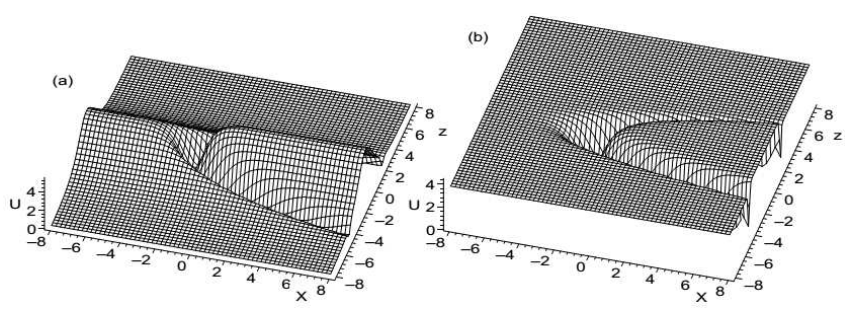

Fig. 1. Intensity plots of the light bullet solitary wave solutions $u_{4}$ expressed by Eq. (30): (a) chirped wave, $a_{0}=0.1$, (b) unchirped wave, $a_{0}=0$, and the parameter selections: $\gamma=\gamma_{0}=0, \beta=1+z, f_{10}=k_{0}=l_{0}=\omega_{0}=$ $b_{0}=R=1, \lambda=0.1$.

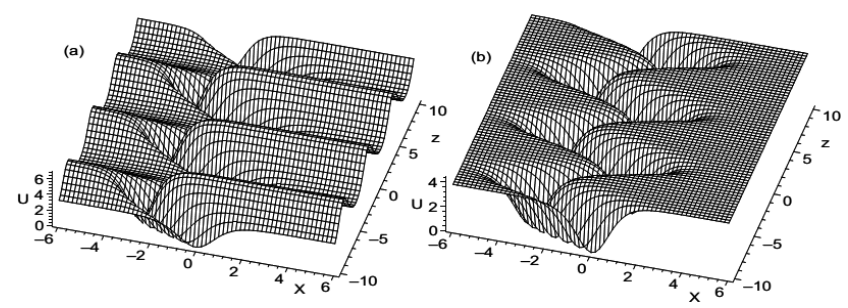

Fig. 2. Intensity plots of the light bullet solitary wave solutions $u_{4}$ expressed by Eq. (30): (a) chirped wave, $a_{0}=0.1,(\mathrm{~b})$ unchirped wave, $a_{0}=0$, and the parameter selections: $\gamma=\gamma_{0}=0, \beta=\cos (z), f_{10}=k_{0}=l_{0}=$ $\omega_{0}=b_{0}=R=1, \lambda=0.1$.

\section{Summary and discussion}

To summarize, applying the generalized $\frac{G^{\prime}}{G}$-expansion method, three types of new exact solutions including the hyperbolic function solution, the trigonometric function solution and the rational solution with parameters for the generalized 2D NLSE with distributed coefficients are obtained. Selecting the parameters and the arbitrary functions in the solutions freely, more abundant exact solutions including soliton solutions can be derived. We present novel exact light bullet soliton solutions with or without the chirp. The influence of the spatiotemporal chirp function on the phase and the amplitude of solutions is displayed. More about the propagation characteristics and its stability for the spatial bullet soliton solution will be investigated somewhere else. The generalized $\frac{G^{\prime}}{G}$-expansion method is extended to solve variable coefficients high-dimensional nonlinear physical models and shown to be effective and powerful. Further study about the solutions for the 2D NLSE and for seeking exact solutions for other higher-dimensional nonlinear models by the generalized $\frac{G^{\prime}}{G}$-expansion method will be worth of investigation.

\section{Acknowledgments}

This work is supported by the Natural Science Foundation of China under grant No. 11272120.

\section{References}

[1] P.L. Christiansen, Future Directions of Nonlinear Dynamics in Physical and Biological System, Plenum Press, New York 1993.

[2] V.E. Zakharov, A.B. Shabat, Sov. Phys. JETP 34, 62 (1971).

[3] D.J. Kaup, Prog. Theor. Phys. 54, 396 (1975).

[4] V.N. Serkin, A. Hasegawa, Phys. Rev. Lett. 85, 4502 (2000).

[5] S. Chen, L. Yi, D.S. Gao, P. Lu, Phys. Rev. E 72, 016622 (2005).

[6] B.E.A. Saleh, M. Teich, Fundamentals of Photonics, Wiley, New York 2007.

[7] M.Z. Rached, E. Recami, J. Math. Phys. 53, 052102 (2012).

[8] I. Towers, B.A. Malomed, J. Opt. Soc. Am. B 19, 537 (2002).

[9] L. Berge, V.K. Mezentsev, J.J. Rasmussen, P.L. Christiansen, Y.B. Gaididei, Opt. Lett. 25, 1037 (2000).

[10] H. Saito, M. Ueda, Phys. Rev. Lett. 90, 040403 (2003).

[11] W.P. Zhong, R.H. Xie, M. Belić, N. Petrović, G. Chen, L. Yin, Phys. Rev. A 78, 023821 (2008).

[12] Y.B. Zhou, M.L. Wang, Y.M. Wang, Phys. Lett. A 308, 31 (2003).

[13] M.L. Wang, X.Z. Li, J.L. Zhang, Phys. Lett. A 372 , 417 (2007).

[14] Y.L. Ma, B.Q. Li, Acta Phys. Sin. 58, 4373 (2009) (in Chinese).

[15] S. Zhang, J.L. Tong, W. Wang, Phys. Lett. A 372, 225 (2008).

[16] W.H. Huang, J.W. Mao, W.G. Qiu, Acta Phys. Pol. A 119, 279 (2011).

[17] V.I. Kruglov, A.C. Peacock, J.D. Harvey, Phys. Rev. E 71, 056619 (2005). 